\title{
Capacitación sobre planeación y evaluación efectivas de proyectos, Unidad de Investigación Científica, Facultad de Ciencias Médicas UNAH, 2008-2018
}

Jackeline Alger ${ }^{1}$

Edna Maradiaga ${ }^{2}$

Aunque la investigación para la salud es el eje de sistemas de salud eficientes y efectivos, se ha descrito que la investigación sigue siendo una débil función de salud pública en las Américas (Etienne, Abbasi, Cuervo, 2018). La realidad es que la organización y gestión de la investigación no existe o tiene un desarrollo muy limitado en los países de la región, a pesar de todos los recursos y herramientas que la era digital ha puesto a la disposición de los individuos y de las instituciones. En la formación de talento humano para la investigación en salud, las instituciones académicas juegan un papel fundamental.

En la Universidad Nacional Autónoma de Honduras (UNAH), la Unidad de Investigación Científica (UIC) es la unidad académica responsable de contribuir al desarrollo de la investigación para la salud en Honduras desde la Facultad de Ciencias Médicas (FCM), a través de la gestión del conocimiento científico, tecnológico, humanístico y ético (Unidad de investigación Científica, Biblioteca Virtual en Salud de Honduras, disponible en http://www.bvs.hn/php/level.php?lang=es\&component=59\&item=11, acceso julio 2018). Durante la década de 2008 al 2018, la UIC y sus aliados estratégicos han contribuido a la formación de talento humano a través de los Cursos de Planeación y Evaluación Efectivas de Proyectos de Investigación (PEEP). Aunque los logros a la fecha son sobresalientes para la UIC, a nivel Facultad se podría haber impulsado más ampliamente los cursos para la construcción de una masa crítica de docentes y administradores formados en buenas prácticas como lo son la planeación y evaluación de los proyectos de investigación para la salud.

\footnotetext{
${ }^{1} \mathrm{MD}, \mathrm{PhD}$, Profesora Titular III, medio tiempo, Unidad de Investigación Científica, Facultad de Ciencias Médicas, Universidad Nacional Autónoma de Honduras, Tegucigalpa, Honduras: jackelinealger@gmail.com

${ }^{2}$ MD, MSP, Profesora Titular III, tiempo completo, Unidad de Investigación Científica, Facultad de Ciencias Médicas, Universidad Nacional Autónoma de Honduras, Tegucigalpa, Honduras.
} 


\section{CAPACITACION SOBRE PLANEACION Y EVALUACION EFECTIVAS DE PROYECTOS}

Recientemente, del 26 al 29 de junio de 2018, la UIC brindó una capacitación más de los Cursos de Planeación y Evaluación Efectivas de Proyectos (PEEP), un curso de Habilidades. Los cursos PEEP son parte de la Iniciativa "Planeando para el éxito" desarrollada por el Programa Especial de Investigación y Capacitación en Enfermedades Tropicales (TDR) de la Organización Mundial de la Salud, UNICEF, PNUD, el Banco Mundial (TDR World Health Organization, disponible en http://www.who.int/tdr/en/, acceso julio 2018) y de cuya red inter-regional es miembro la UIC. Para Latinoamérica, el Centro Regional de Capacitación del TDR es CIDEIM (Centro Internacional de Entrenamiento e Investigación Médica), localizado en Cali, Colombia (CIDEIM, disponible en http://www.cideim.org.co/cideim/; acceso julio 2018) (Tulloch-Reid et al, 2018; WHO, 2018a). A través de la relación con CIDEIM y con apoyo de la Organización Panamericana de la Salud/Organización Mundial de la Salud, la UIC conoció y comenzó a construir capacidad en planeación y evaluación de proyectos en el año 2008 cuando tres docentes de la UIC fueron invitados y capacitados en las habilidades básicas mediante un Curso PEEP desarrollado por CIDEIM.

El proceso de capacitación de los cursos PEEP se ha sistematizado por parte de TDR OMS y cuenta con una serie de recursos y herramientas para la capacitación en habilidades básicas (Curso PEEP Habilidades, Skill-Building Course) y el curso de capacitación de capacitadores (Train The Trainer Course, Curso PEEP TTT) (WHO, 2018b). Cuenta con manuales, diapositivas, lineamientos y modelos de los diferentes procesos y documentos que se utilizan en la organización de los cursos (lista de cotejo de la organización, correspondencia, certificados, compromiso de conducta ética, evaluaciones, curso virtual de repaso para los capacitadores, entre otros). El curso se imparte en 28 horas presenciales distribuidas en cuatro días y requiere unas 8 horas de trabajo individual adicional. Los participantes que adquieren las habilidades básicas y que así lo deseen, que además cuenten con características para capacitar a otros y con el apoyo institucional, después de un tiempo de aplicar el conocimiento y las herramientas PEEP adquiridos, pueden ser capacitados como capacitadores y de esa manera contribuir a institucionalizar la iniciativa. El curso PEEP TTT también se imparte de manera presencial en cuatro días. Los cursos PEEP buscan fortalecer las habilidades de investigadores y profesionales de la salud en las áreas de planeación, organización, gestión y evaluación de sus proyectos, para así asegurar una implementación exitosa, colaboración efectiva, y la competitividad para acceder a financiamiento internacional (Alger, 2010). 
La metodología aplicada en los cursos PEEP es la de "aprender haciendo". Los participantes trabajan en grupos de tres personas, cuatro grupos en total. Es decir, 12 participantes en total. Cada grupo trabaja con su propio proyecto durante un $80 \%$ del tiempo del curso lo cual les permite a los participantes aplicar los pasos de planeación y evaluación a su proyecto. El Curso se desarrolla a través de cinco módulos. Módulo 1, Buenas prácticas en la investigación biomédica (teoría); Módulo 2, Entender el concepto y valor de la planeación y evaluación de proyectos (teoría); Módulo 3, Fase 1: Definir el propósito y alcance del proyecto (teoría y estudio de casos); Módulo 4, Fase 2: Establecer el plan de desarrollo del proyecto (teoría y estudio de casos); Módulo 5, Fase 3: Ejecución, supervisión, evaluación y reporte (teoría y estudio de casos) (Ver Figura 1). Es así como los grupos que asisten al curso aplican la metodología PEEP a sus propios proyectos, definiendo primero el propósito y alcance de estos, luego desarrollando un plan de trabajo y finalmente estableciendo parámetros de comunicación que faciliten la implementación, el seguimiento y la evaluación de actividades. Esto se logra proporcionando las herramientas necesarias para la planeación y evaluación de proyectos. Al final del curso, los grupos habrán desarrollado una versión inicial de la documentación de su proyecto, la cual incluirá una definición clara del proyecto, objetivos específicos y medibles, un plan de trabajo realista para alcanzar los objetivos establecidos (incluyendo actividades para ser realizadas, cronologías, personas responsables, presupuesto, etc.), descripción de los miembros del equipo del proyecto, con su experiencia y responsabilidades correspondientes identificadas y una estructura para el trabajo en equipo que facilitará la comunicación y la implementación del proyecto (WHO, 2018b). La metodología se puede aplicar a proyectos de investigación y también a proyectos de desarrollo institucional.

El primer curso PEEP Habilidades impartido en Honduras fue organizado en el año 2008. El curso desarrollado en el año 2018, diez años después, es el quinto Curso PEEP Habilidades impartido. Esta pausa en el desarrollo de los cursos se debe a que en el periodo 2009-2012 no se impartió ningún curso, aunque se realizaron las gestiones administrativas desde la UIC. Las instituciones hondureñas que han participado incluyen a la FCM UNAH (UIC, POSAP, Escuela de Enfermería, Coordinación de Postgrados, Carrera de Nutrición, Carrera de Terapia Funcional), Secretaría de Salud e Instituto Hondureño de Seguridad Social (ver Cuadro 1). En cuatro de los cinco cursos PEEP Habilidades desarrollados, se ha contado con participantes de los países centroamericanos. Los países participantes han sido Guatemala $(2008,2013$, 2018), Costa Rica (2014), El Salvador (2014). También se han realizado dos cursos PEEP TTT con la participación de Cuba (2008) y Guatemala (2014). Por lo tanto, Honduras se ha convertido en líder en la diseminación de la iniciativa "Planeando para el Éxito" en la sub-región de Centro América. 
Figura 1. Proceso de planeación y evaluación efectivas de proyectos de investigación para la salud través de cinco módulos y tres fases

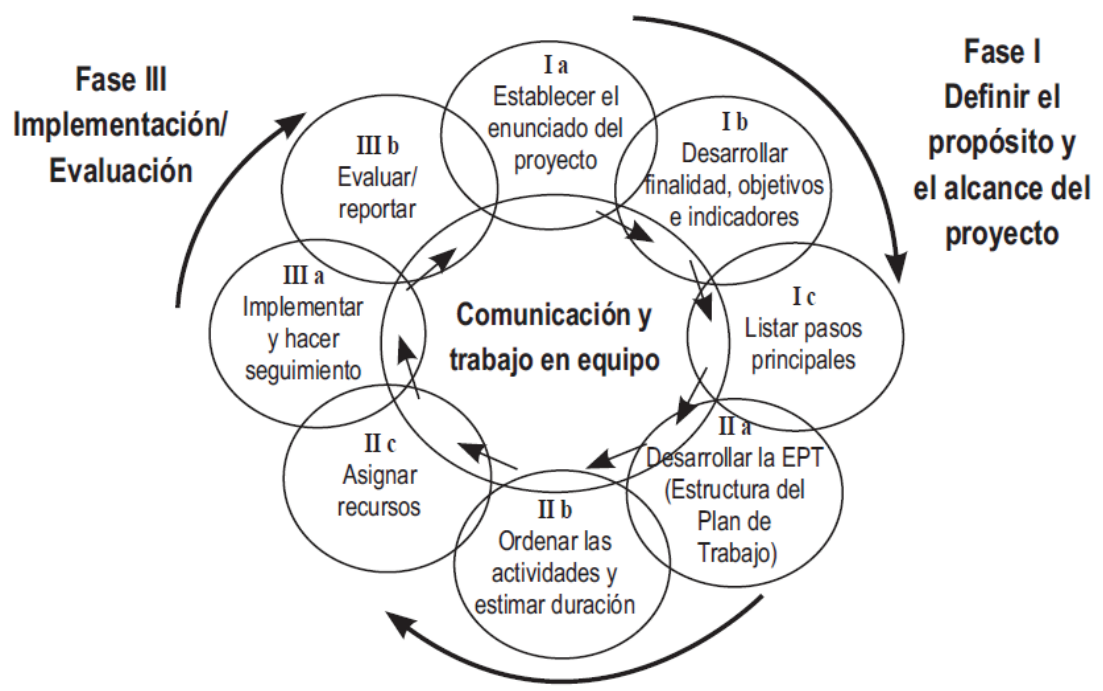

Fase II: Planeación

Fuente: Guía Paso a Paso. Planeación y Evaluación Efectivas de Proyectos de Investigación Biomédica. TDR OMS 2008

En cuanto a la capacitación de capacitadores, se ha formado a capacitadores en UIC (4), Maestría Salud Pública (1) y Escuela de Enfermería (2), un total de siete en la FCM UNAH. De estos, en la actualidad se cuentan con cuatro capacitadores que han impartido al menos un curso PEEP Habilidades, dos en UIC y dos en Escuela de Enfermería. Una de las capacitadoras tiene la experiencia de haber participado en los cinco cursos impartidos en Honduras y dos cursos internacionales (Paramaribo, Surinam y Medellín, Colombia). En varios de los cursos impartidos en Honduras, se ha contado con apoyo en la facilitación por parte de profesionales internacionales; Fiocruz, Brasil (2008) y CIDEIM, Colombia $(2013,2014)$. En los años 2017 y 2018, los cursos PEEP Habilidades fueron impartidos en su totalidad por facilitadoras hondureñas, demostrando el apoyo institucional desde UIC y Escuela de Enfermería a esta iniciativa. Adicionalmente, un importante logro para la integración de la iniciativa en la FCM UNAH es la adaptación del curso PEEP Habilidades como un Módulo de Gestión de Proyectos en el programa académico de la Maestría en Salud Pública (POSAP), capacitando a la fecha dos cohortes de maestrandos en los años 2014 y 2016, a través de una coordinación efectiva entre los docentes POSAP y la UIC. A la fecha, en Honduras se ha capacitado un total 125 profesionales en habilidades, incluyendo 13 de la subregión de Centro América, y 8 profesionales como capacitadores, 
3 de la sub-región de Centro América y el Caribe (Ver Cuadro 1). Para completar todas estas actividades se ha requerido la gestión técnico-administrativa desde la UIC y la inclusión de los Cursos PEEP en el Plan Operativo Anual.

Cuadro 1. Numero de capacitados en cursos de Planeación y Evaluación Efectivas de Proyectos en Habilidades y como Capacitadores, Cursos impartidos en Honduras, Facultad de Ciencias Médicas UNAH, 2008-2018.

\begin{tabular}{|c|c|c|}
\hline PAIS / INSTITUCION & $\begin{array}{l}\text { CAPACITADOS EN } \\
\text { HABILIDADES }{ }^{1}\end{array}$ & CAPACITADORES 2 \\
\hline HONDURAS & 115 & $7^{*}$ \\
\hline Facultad de Ciencias Médicas & 109 & 7 \\
\hline Unidad de Investigación Científica & 14 & 4 \\
\hline Escuela de Enfermería & 18 & 2 \\
\hline Maestría en Salud Pública** & 69 & 1 \\
\hline Coordinación General de Postgrados & 3 & 0 \\
\hline Carrera de Nutrición & 2 & 0 \\
\hline Carrera de Terapia Física & 3 & 0 \\
\hline Secretaría de Salud & 1 & 0 \\
\hline $\begin{array}{l}\text { Instituto Hondureño de Seguridad } \\
\text { Social }\end{array}$ & 5 & 0 \\
\hline GUATEMALA & 7 & 2 \\
\hline COSTA RICA & 3 & 0 \\
\hline EL SALVADOR & 3 & 0 \\
\hline CUBA & 0 & 1 \\
\hline TOTAL & 128 & 10 \\
\hline \multicolumn{3}{|c|}{$\begin{array}{l}\text { 1Tres capacitados fuera de Honduras; }{ }^{2} \text { Dos capacitados fuera de Honduras; }{ }^{*} \text { Activos, } n=4 ; \\
\text { **Dos cohortes de maestrandos capacitados en habilidades mediante Módulo de Gestión de } \\
\text { Proyectos, } 2014(n=36), 2016(n=28) \text {. }\end{array}$} \\
\hline
\end{tabular}

Fuente: Elaboración propia.

\section{CONCLUSIONES}

En la actualidad, la FCM UNAH cuenta con un número importante de docentes capacitados en habilidades, distribuidos en diferentes unidades académicas, y quienes han expresado su interés en ser capacitados como capacitadores. Sería estratégico para la FCM UNAH dirigir los esfuerzos y recursos para realizar cursos PEEP TTT que permitirán contar con un mayor número de capacitadores. Esta masa crítica de 
docentes capacitados y capacitadores contribuirá a institucionalizar los cursos PEEP y fortalecer la capacidad de formular proyectos de investigación, su implementación exitosa, la colaboración efectiva y aumentar la competitividad para acceder a financiamiento internacional. Consideramos que la iniciativa "Planeando para el Éxito", los cursos PEEP y el trabajo en red con CIDEIM, son facilitadores y potenciadores para la construcción de dicha capacidad.

\section{REFERENCIAS BIBLIOGRÁFICAS}

Alger J, L Gómez, A Jaramillo, NG Saravia, LG Cuervo, B Halpaap. (2010). Red Inter-Regional de planeación y Evaluación Efectivas de Proyectos de Investigación para la Salud, Cali, Colombia, Abril 2010. Revista Médica Hondureña 78: 96-99.

CIDEIM, Programa Especial de Investigaciones y Enseñanzas sobre Enfermedades Tropicales (TDR), Organización Panamericana de la Salud, Organización Mundial de la Salud. (2008). Planeación y Evaluación Efectivas de Proyectos de Investigación Biomédica. Manual de Entrenamiento. Participantes. Organización Mundial de la Salud, Ginebra, Suiza.

Etienne C, Abbasi K, Cuervo LG. (2018). Research for health in the Americas. British Medical Journal 362:k2944. Disponible en https://www.bmj.com/content/bmj/362/bmj.k2944.fuII.pdf

Tulloch-Reid MK, Gore Saravia N, Dennis RJ, Jaramillo A, Cuervo LG, Walker SP, Salicrup LA. (2018). Strengthening institutional capacity for equitable health research: lessons from Latin America and the Caribbean. British Medical Journal 362:k2456. Disponible en https://www.bmj.com/content/bmj/362/bmj.k2456.full.pdf.

World Health Organization. (2018a). Regional Training Centres. http://www.who.int/tdr/capacity/strengthening/regional/en/.

World Health Organization (2018b). Effective project planning and evaluation for biomedical and health research. Disponible en http://www.who.int/tdr/publications/topics/project_planning_training/en/. 